\title{
Contribution of submandibular gland and swallowing structure sparing to post- radiation therapy PEG dependence in oropharynx cancer patients treated with split-neck IMRT technique
}

\author{
Michael F. Gensheimer', Matthew Nyflot ${ }^{2}$, George E. Laramore ${ }^{2}$, Jay J. Liao ${ }^{2}$ and Upendra Parvathaneni2 ${ }^{2 *}$
}

\begin{abstract}
Background: Radiation therapy-related dysphagia is worsened by xerostomia. The submandibular glands (SMG) produce saliva rich in lubricating mucins, and sparing the SMG has been shown to reduce xerostomia. The goal of this study was to determine whether SMG sparing IMRT is associated with reduced post-treatment PEG dependence in locally advanced oropharynx cancer patients.

Methods: Patients treated with definitive radiation therapy for oropharynx cancer were included in this retrospective study. Those with disease recurrence were excluded. Salivary glands and swallowing-related organs at risk, including pharyngeal constrictors, were contoured. Primary endpoint was time from end of radiation treatment to freedom from gastrostomy (PEG) tube dependence. Cox proportional hazards regression and logistic regression were used to assess influence of normal tissue doses on swallowing related endpoints.

Results: Sixty-nine patients were included. All had stage III/IV disease and 97\% received concurrent systemic therapy. Fifty-seven percent had contralateral SMG (CSMG) mean dose <50 Gy, a level shown to predict for xerostomia. Eighty four percent of patients had a PEG tube placed electively. On univariate analysis, the strongest predictor of time to freedom from PEG tube dependence was CSMG dose (HR 0.97 per Gy (95\% Cl 0.95-0.98), $p<0.0001$ ). This relationship persisted on multivariate analysis $(p=0.052)$. The dose to superior and middle pharyngeal constrictor muscles, and larynx were also significant on univariate analysis. Patients with cSMG dose less than median (42 Gy, $n=34$ ) had a significantly shorter time to freedom from PEG dependence: median 1.9 vs. 3.5 months, $p<0.0001$. At 6 months, $3 \%$ of patients with cSMG dose < 42 Gy were PEG dependent compared to 31\% with cSMG dose > 42 Gy $(p=0.002)$.

Conclusions: Patients treated with CSMG sparing radiotherapy had significantly shorter time to PEG tube removal after treatment, suggesting a clinically meaningful reduction in subacute dysphagia compared to non-CSMG sparing treatment.
\end{abstract}

Keywords: Head and neck cancer, Oropharynx, Xerostomia, Dysphagia, Radiotherapy, Submandibular gland

\footnotetext{
*Correspondence: upendra@uw.edu; upendra@u.washington.edu

${ }^{2}$ Department of Radiation Oncology, University of Washington Medical

Center, 1959 NE Pacific St Box 356043, Seattle, WA 98195, USA

Full list of author information is available at the end of the article
} 


\section{Background}

Dysphagia is a common late toxicity of radiation therapy for head and neck cancer and is detrimental to quality of life $[1,2]$. A variety of risk factors for post-treatment dysphagia have been identified, including use of concurrent chemotherapy, advanced disease stage, and higher dose to pharyngeal constrictor muscles and other swallowing-related organs [3-5]. However, retrospective studies have shown little consistency in which individual swallowing-related structures are most important, and some studies failed to show a correlation between pharyngeal constrictor muscle dose and dysphagia when controlling for tumor location and other factors $[1,6,7]$. In a prospective study in oropharynx cancer patients, even when treatment was planned to minimize dose to swallowing structures (pharyngeal constrictors, larynx, and esophageal inlet), post-treatment dysphagia was common and persisted years after treatment [2]. Together, these data indicate a need for additional strategies to minimize post-treatment dysphagia.

Another common side effect of head and neck radiation therapy, xerostomia, exacerbates post-treatment dysphagia. Xerostomia is known to impede the normal swallowing process, especially for dry foods. In a study of post-radiation therapy patients with xerostomia, the swallowing steps of mastication and oral manipulation were compromised for solid foods [8]. Other studies have shown that post-treatment dysphagia and xerostomia are highly correlated, for both observer- and patient-reported measures [9-11]. Therefore, sparing the function of the salivary glands and reducing xerostomia could be a contributory strategy to reduce treatment-induced dysphagia. However, studies have not shown a consistent link between sparing the parotid gland alone and reducing dysphagia. In the PARSPORT randomized study comparing parotid sparing versus non-salivary sparing radiation therapy in head and neck cancer patients, late grade 3-4 dysphagia was actually more common in parotid spared patients at 12 months (9\% vs. 5\%) [12]. Sparing one or both submandibular glands (SMG) in addition to sparing the parotid glands has been shown to further reduce xerostomia in patients with oropharynx cancer [13, 14]. The SMGs produce saliva rich in mucins that act as a lubricant during the swallowing process [15]. While stimulated saliva is largely derived from the parotid, the optimal viscosity of the food bolus required for a successful swallow is also dependent on SMG derived mucinous saliva [15-18]. The goal of the present study was to determine whether contralateral submandibular gland (cSMG) sparing treatment is associated with decreased duration of PEG (percutaneous endoscopic gastrostomy) tube dependence, an objective endpoint that reflects subacute dysphagia.

\section{Methods}

\section{Patient population}

This was a retrospective study approved by the Institutional Review Board. Eligible patients were treated at our institution for oropharyngeal squamous cell carcinoma with definitive IMRT including bilateral neck treatment. Patients were required to have six months or longer follow-up in order to assess late toxicities. Those treated prior to late 2007 were not eligible because their computerized treatment plans were no longer accessible. Exclusion criteria included primary tumor resection prior to radiation therapy, any disease recurrence after radiation therapy, and prior radiation therapy to the head and neck region.

\section{Feeding tube placement}

Per institutional policy, patients receiving concurrent chemoradiation had PEG tubes placed prophylactically prior to starting treatment unless they declined this procedure. For the patients who received radiation therapy without chemotherapy a case-by-case determination on prophylactic vs. as-needed (reactive) placement is made. After treatment, the feeding tube is removed once the patient is able to maintain his/her weight for $1-2$ weeks using oral nutrition alone. These policies have not changed over the study period.

\section{Radiotherapy technique}

The radiation therapy parameters used for these patients have been detailed previously [13]. In general, patients with primary tumor not encroaching on the cSMG and without contralateral nodal involvement had cSMG sparing treatment; in the other patients no attempt was made to reduce cSMG dose. When possible, bilateral parotid glands were spared to achieve mean dose < $24 \mathrm{~Gy}$. In order to reduce larynx dose, a split field technique was used with a matched low-neck en face field with a larynx block. No specific attempt was made to spare the pharyngeal constrictor muscles, though there was an IMRT avoidance structure consisting of the oral cavity/oropharynx outside of the PTV.

A simultaneous integrated boost method was used. The highest risk target volume (PTV1) was generally prescribed 70 Gy in 33 fractions; the intermediate and low risk subclinical volumes (PTV2 and PTV3) received 62.7 and $57 \mathrm{~Gy}$, respectively.

\section{Analysis}

The primary endpoint was time from end of radiation treatment to freedom from PEG tube dependence. Patients who never required gastrostomy tube were scored as achieving the endpoint at time 0. The Kaplan-Meier method was used to estimate time to PEG tube removal. Patients were censored at time of last follow-up or 
death. Impact of clinical and dosimetric factors on this endpoint was assessed using univariate and multivariate Cox proportional hazards regression.

The secondary endpoint was presence/absence of grade $\geq 2$ late (6 months or more after treatment completion) observer-rated dysphagia as defined by the Common Terminology Criteria for Adverse Events (CTCAE) version 4 [19]. Univariate and multivariate logistic regression was used to identify patient factors associated with the outcome. For multivariate analysis of both endpoints, only covariates with significant $p$ values $(p<0.05)$ on univariate analysis were included due to sample size limitations.

Normal structures, including salivary glands and swallowing structures, were contoured for the study by one physician (M.G.) using established guidelines [20]. To improve the uniformity and efficiency of segmentation, atlas-based contouring was used in MIM version 6 (MIM Software, Cleveland, Ohio, USA). Contours were drawn on five patients' CTs and propagated to the rest of the patients using deformable registration. Contours were then manually edited to ensure consistency and accuracy.

For the dosimetric analysis, we included the swallowing structures found to be significantly correlated with dysphagia in prior studies: the superior, middle, and inferior constrictor muscles, cricopharyngeal muscle, esophageal inlet muscles, and glottic/supraglottic larynx $[1,3,5]$. We also included the salivary structures: cSMG, parotid glands, and oral cavity (surrogate for minor salivary glands). For SMG dose, only the contralateral gland was used since the ipsilateral gland always overlapped the high dose planning target volume and would not be expected to have preserved function after treatment. For parotid gland dose, the mean of the two parotid gland doses was used since both parotid glands were usually spared and had similar doses. For all structures, only mean dose was used, because for both swallowing structures and salivary glands mean dose has been found to be highly predictive of patient outcomes [3, 21].

Differences in CSMG dose between patients with various clinical characteristics were assessed using the Wilcoxon rank-sum test. Statistical calculations were done with R software, version 3 (University of Auckland).

\section{Results}

\section{Patients and treatments}

Sixty-nine patients treated between Dec. 2007 and Feb. 2014 met the inclusion criteria. Patient characteristics are listed in Table 1. Almost all patients had stage IV disease (91\%) and received concurrent systemic therapy (97\%). The most common systemic therapy was highdose cisplatin $(n=55)$.
Table 1 Patient characteristics

\begin{tabular}{|c|c|}
\hline Characteristic & Number (\%) \\
\hline \multicolumn{2}{|l|}{ Age at treatment } \\
\hline Mean & 57 \\
\hline Range & $25-73$ \\
\hline \multicolumn{2}{|c|}{ Concurrent systemic therapy } \\
\hline Yes & $67(97 \%)$ \\
\hline No & $2(3 \%)$ \\
\hline \multicolumn{2}{|l|}{ Disease subsite } \\
\hline Tonsil & $32(46 \%)$ \\
\hline Base of tongue & $30(43 \%)$ \\
\hline Other/multiple & $7(10 \%)$ \\
\hline \multicolumn{2}{|l|}{ AJCC stage } \\
\hline III & $6(9 \%)$ \\
\hline IVA & $50(72 \%)$ \\
\hline IVB & $13(19 \%)$ \\
\hline \multicolumn{2}{|l|}{ T stage } \\
\hline 1 & $5(7 \%)$ \\
\hline 2 & $19(28 \%)$ \\
\hline 3 & $17(25 \%)$ \\
\hline $4 a$ & $21(30 \%)$ \\
\hline $4 b$ & $7(10 \%)$ \\
\hline \multicolumn{2}{|l|}{$N$ stage } \\
\hline 0 & $2(3 \%)$ \\
\hline 1 & $7(10 \%)$ \\
\hline $2 a$ & $5(7 \%)$ \\
\hline $2 b$ & $27(39 \%)$ \\
\hline $2 c$ & $23(33 \%)$ \\
\hline 3 & $5(7 \%)$ \\
\hline
\end{tabular}

Dose to the primary tumor ranged from 66 to $72 \mathrm{~Gy}$; 64 of 69 patients received a dose of 69.96 Gy in 33 fractions. Normal structure doses are listed in Table 2. In forty-three patients there was an IMRT objective for the cSMG. No patient had an IMRT objective for the

Table 2 Doses received by normal structures

\begin{tabular}{ll}
\hline Structure & Mean dose in Gy (range) \\
\hline Contralateral submandibular gland & $45.23(18.16-74.24)$ \\
Parotid glands (bilateral) & $32.50(18.90-50.92)$ \\
Superior pharyngeal constrictor & $61.05(39.54-70.85)$ \\
Middle pharyngeal constrictor & $62.74(41.57-72.08)$ \\
Inferior pharyngeal constrictor & $41.83(21.90-70.04)$ \\
Esophageal inlet muscles & $36.37(10.23-62.91)$ \\
Cricopharyngeal muscle & $30.85(9.86-69.08)$ \\
Glottic/supraglottic larynx & $46.70(23.91-69.89)$ \\
Oral cavity & $41.41(17.37-72.00)$ \\
\hline
\end{tabular}


ipsilateral SMG. Thirty-nine patients (57\%) had cSMG mean dose $<50 \mathrm{~Gy}$, a level which has been shown to predict preservation of gland function [21]. We examined whether baseline patient/tumor factors were associated with ability to perform cSMG sparing. Patients with bilateral nodal disease had higher cSMG doses than others (mean 59.3 vs. 36.7 Gy, $p<0.0001$ ). Tumor stage (T1-3 vs. T4) and primary site (base of tongue vs. other) were not significantly associated with cSMG dose.

\section{Toxicity outcomes}

Median follow-up was 27 months (range 7-78 months). Consistent with the inclusion criteria, no patient suffered disease recurrence. Two patients are deceased; cause of death was endocarditis in one and unknown in the other.

Fifty-eight patients (84\%) had a gastrostomy tube placed prior to or during radiation therapy. Seventy-nine percent of these were placed before or during the first week of treatment, consistent with our department policy of prophylactic feeding tube placement for patients receiving chemoradiation. No patient had a feeding tube placed after completion of radiation therapy. In patients with a gastrostomy tube placed, median time to tube removal was 3.2 months. Gastrostomy tube dependence rate was $17 \%$ six months after treatment and $4 \%$ at twelve months. Two patients were tube dependent at last follow-up, 11 and 37 months after treatment.

Predictors of time to freedom from PEG dependence were examined using Cox proportional hazards regression (Table 3). On univariate analysis, the strongest predictor was cSMG dose (HR 0.97 per Gy, $p<0.0001$ ). Other significant predictors were dose to the superior and middle pharyngeal constrictors, esophageal inlet muscles, larynx, and oral cavity. Parotid dose was not significant on any analysis. On multivariate analysis, cSMG dose was still the strongest predictor of this endpoint (HR 0.98 per Gy, $p=0.052$ ). Median cSMG dose was 42.3 Gy. Patients with cSMG dose less than median $(n=34)$ had significantly shorter time to freedom from PEG tube dependence: median 1.9 vs. 3.5 months, $p<0.0001$ by log-rank test (Fig. 1). At 6 months, only $3 \%$ (95\% CI $0.4-20.2 \%$ ) of patients with cSMG dose less than median were PEG tube dependent, versus $31 \%$ (95\% CI 19.3-51.3\%) of those with cSMG dose greater than median $(p=0.002)$. None of the patients with cSMG dose less than median were PEG tube dependent at 12 months.

The distribution of observer-rated late dysphagia (worst grade six months or later after treatment, CTCAE version 4) was: grade 0: 44 patients; grade 1: 10 patients; grade 2: 6 patients; grade 3: 9 patients. We examined predictors of late grade $\geq 2$ dysphagia, defined as symptomatic and altered eating/swallowing (Table 4). On univariate analysis, cSMG dose was the strongest predictor of late dysphagia (OR 1.06 per Gy, $p=0.004$ ). Other significant predictors were T4 vs. T1-3 tumor stage, bilateral nodal disease, superior constrictor dose, and oral cavity dose. On multivariate analysis, the only significant predictor of late dysphagia was T4 tumor stage (OR 4.60, $p=0.04$ ).

Thirty-nine patients $(57 \%)$ had grade $\geq 2$ observerrated late xerostomia. Consistent with our prior report, patients with higher CSMG dose were more prone to grade $\geq 2$ late xerostomia (OR 1.05 per $\mathrm{Gy}, p=0.002$ ) [13]. Oral cavity dose was also associated with late xerostomia (OR 1.05 per Gy, $p=0.05$ ), but parotid gland dose was not $(p=0.51)$. Observer-rated late dysphagia and xerostomia were modestly correlated, with a phi coefficient of $0.32, p=0.02$ by the chi-square test.

Table 3 Predictors of time to freedom from PEG tube dependence (Cox proportional hazards model). Lower hazard ratio indicates later PEG tube removal

\begin{tabular}{|c|c|c|c|c|}
\hline \multirow[t]{2}{*}{ Variable (mean doses in Gy) } & \multicolumn{2}{|l|}{ Univariate } & \multicolumn{2}{|l|}{ Multivariate } \\
\hline & Hazard ratio $(95 \% \mathrm{Cl})$ & $P$ value & Hazard ratio $(95 \% \mathrm{Cl})$ & $P$ value \\
\hline Contralateral submandibular gland dose & $0.97(0.95-0.98)$ & $<0.0001$ & $0.98(0.95-1.00)$ & 0.052 \\
\hline Middle pharyngeal constrictor dose & $0.92(0.88-0.96)$ & 0.0002 & $0.95(0.89-1.02)$ & 0.18 \\
\hline Superior pharyngeal constrictor dose & $0.95(0.92-0.98)$ & 0.003 & $0.98(0.93-1.03)$ & 0.38 \\
\hline Glottic/supraglottic larynx dose & $0.97(0.94-0.99)$ & 0.007 & $1.02(0.98-1.07)$ & 0.40 \\
\hline Esophageal inlet muscles dose & $0.98(0.97-1.00)$ & 0.03 & $0.99(0.97-1.01)$ & 0.39 \\
\hline Oral cavity dose & $0.97(0.95-0.99)$ & 0.03 & $1.00(0.97-1.04)$ & 0.86 \\
\hline Inferior pharyngeal constrictor dose & $0.98(0.96-1.00)$ & 0.09 & & \\
\hline T4 tumor stage & $0.69(0.42-1.15)$ & 0.16 & & \\
\hline Parotid glands mean dose & $0.98(0.94-1.02)$ & 0.24 & & \\
\hline Bilateral nodal involvement & $0.77(0.47-1.27)$ & 0.31 & & \\
\hline Cricopharyngeal muscle dose & $0.99(0.97-1.01)$ & 0.34 & & \\
\hline Age & $1.00(0.97-1.03)$ & 0.96 & & \\
\hline
\end{tabular}

Statistically significant values are given in bold 


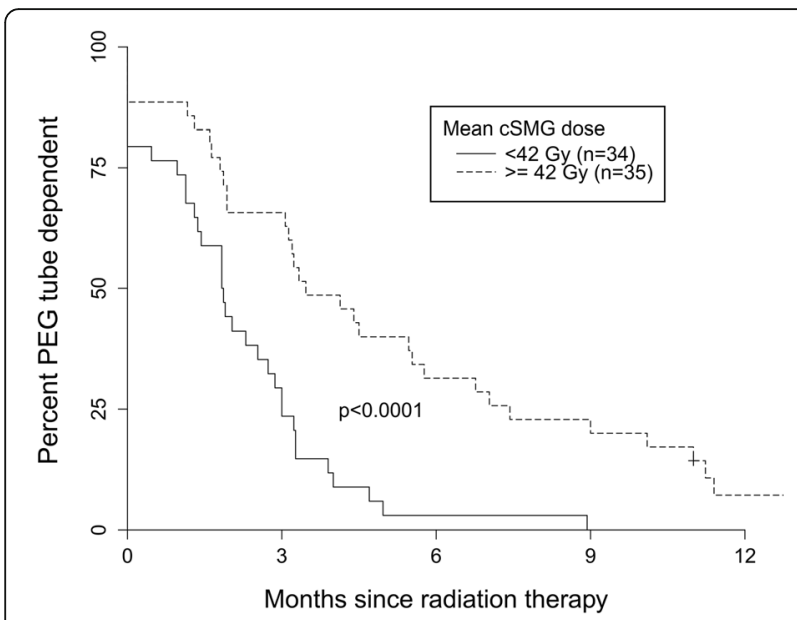

Fig. 1 Freedom from PEG tube dependence by mean contralateral submandibular gland dose greater or less than median

\section{Discussion}

We examined the influence of clinical and dosimetric factors on post-treatment PEG dependence in patients with locally advanced oropharynx cancer. cSMG dose was the strongest predictor of time to freedom from PEG tube dependence on both univariate and multivariate analysis. Patients with cSMG dose less than median (42 Gy) had a 3\% rate of PEG tube dependence at 6 months and $0 \%$ at 12 months. To our knowledge, this is the first report of an objectively measured dysphagia related outcome, i.e., post treatment PEG dependence, after cSMG sparing bilateral neck IMRT.

Most studies correlating radiation dose to pharyngeal constrictor muscles and other swallowing-related organs (larynx, and esophageal inlet) did not include the salivary glands in their models of dysphagia [3, 6, 7]. During the process of swallowing a food bolus, in addition to the neuro-muscular structures involved, an optimal volume and viscosity of saliva, and adequate lubrication are necessary [8, 15-18]. While parotid derived stimulated saliva contributes to the volume of saliva, SMG derived saliva rich in mucins provides the viscosity and lubrication needed for swallowing of foods such as dry solids [15]. A prospective study of 93 patients treated with radiation therapy showed that post-treatment patientreported xerostomia grade was a strong predictor of dysphagia grade [9]. Thus, it is plausible that minimizing xerostomia, by preserving the function of the salivary glands in addition to sparing the muscular swallowing structures, could reduce post-treatment dysphagia and PEG dependence.

Our analysis suggests that sparing the cSMG could be an important factor in minimizing detriment to the swallowing process. This is also supported by a study of 354 patients with mostly oropharynx and larynx primaries showing that SMG doses were significant predictors of post-treatment swallowing dysfunction on univariate analysis [5]. While parotid glands were routinely spared (in addition to cSMG) in our cohort, sparing the parotid glands alone is unlikely to improve swallowing function as parotid saliva is watery and lacks mucins [12].

The two endpoints in this study (freedom from gastrostomy tube dependence after treatment, and late observerrated late dysphagia) have both been commonly used as indicators of dysphagia after radiation therapy [2, 4, 22]. The results for the two endpoints differed somewhat. While cSMG dose was predictive of both endpoints on univariate analysis, on multivariate analysis, cSMG dose was a strong predictor of the objective gastrostomy tube endpoint but not observer-rated dysphagia $(p=0.052$, 0.29 , respectively). The feeding tube endpoint may be more relevant to patients, for a few reasons. First, duration

Table 4 Predictors of grade $\geq 2$ late observer-rated dysphagia (logistic regression)

\begin{tabular}{|c|c|c|c|c|}
\hline \multirow[t]{2}{*}{ Variable (mean doses in Gy) } & \multicolumn{2}{|l|}{ Univariate } & \multicolumn{2}{|l|}{ Multivariate } \\
\hline & Odds ratio $(95 \% \mathrm{Cl})$ & $P$ value & Odds ratio $(95 \% \mathrm{Cl})$ & $P$ value \\
\hline Contralateral submandibular gland dose & $1.06(1.02-1.10)$ & 0.004 & $1.03(0.98-1.09)$ & 0.29 \\
\hline T4 tumor stage & $5.99(1.77-24.18)$ & 0.006 & $4.60(1.17-21.16)$ & 0.04 \\
\hline Superior pharyngeal constrictor dose & $1.27(1.09-1.56)$ & 0.009 & $1.15(0.98-1.42)$ & 0.12 \\
\hline Oral cavity dose & $1.08(1.01-1.16)$ & 0.03 & $1.02(0.94-1.12)$ & 0.62 \\
\hline Bilateral nodal involvement & $3.26(1.02-11.18)$ & 0.05 & $1.45(0.28-7.56)$ & 0.65 \\
\hline Glottic/supraglottic larynx dose & $1.06(1.00-1.12)$ & 0.06 & & \\
\hline Parotid glands mean dose & $1.06(0.99-1.15)$ & 0.11 & & \\
\hline Inferior pharyngeal constrictor dose & $1.04(0.99-1.09)$ & 0.13 & & \\
\hline Middle pharyngeal constrictor dose & $1.08(0.98-1.21)$ & 0.15 & & \\
\hline Cricopharyngeal muscle dose & $1.02(0.98-1.07)$ & 0.35 & & \\
\hline Age & $1.02(0.95-1.10)$ & 0.56 & & \\
\hline Esophageal inlet muscles dose & $1.01(0.97-1.05)$ & 0.63 & & \\
\hline
\end{tabular}


of feeding tube requirement correlates with reduced quality of life related to late dysphagia. In a study of head and neck cancer patients one year after treatment, having a feeding tube present at one year was associated with reduced quality of life in nine of twelve subscales [23]. Our patients' 4\% PEG tube dependence rate at 12 months is similar to the $1 \%$ rate reported in the constrictor sparing trial [2] and less than the 9\% rate reported among stage III-IV patients receiving chemoradiation in a multiinstitutional study [4].

Second, observer-rated dysphagia is a subjective endpoint and is known to correlate poorly with patientreported dysphagia [24]. For instance, in a recent prospective study only $1 \%$ of patients had grade 2 or worse observer-scored dysphagia 24 months after radiation therapy, compared to over $50 \%$ of patients reporting dysphagia at the same time point [9].

An interesting issue could be the tradeoff between cSMG sparing and pharyngeal constrictor sparing. The submandibular glands are located at a similar craniocaudal location as the middle pharyngeal constrictor muscle (Fig. 2). A potential concern is that by aggressively sparing the CSMG instead of prioritizing pharyngeal constrictor sparing, the dose to swallowing muscles

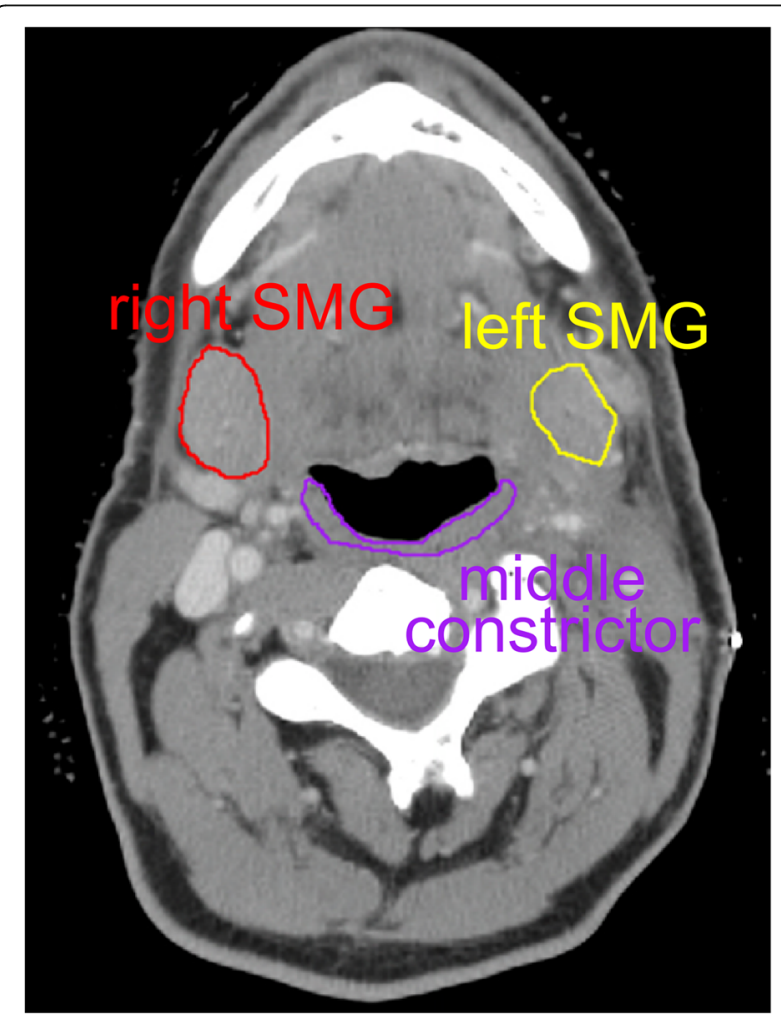

Fig. 2 Relationship of submandibular glands to middle pharyngeal constrictor muscle. There may be a tradeoff in radiation treatment planning between aggressively sparing the constrictor muscles and the contralateral submandibular gland could increase, worsening dysphagia. A crude comparison shows that our patients' swallowing structure doses are similar to those in a trial of constrictor muscle sparing IMRT in which the mean constrictor dose was $58 \mathrm{~Gy}$, the mean larynx dose was $48 \mathrm{~Gy}$, and the mean esophageal inlet dose was 34 Gy [2]. This suggests that cSMG sparing in our series did not overly increase dose to the swallowing structures, as we routinely spared the uninvolved oral cavity and oropharynx.

The main limitation of this study is the non-randomized nature of the CSMG sparing treatment, raising the possibility of bias and unmeasured confounders. Patients without bilateral nodal involvement were more likely to have cSMG sparing; nodal stage has been shown in some other series to be a predictor of post-treatment dysphagia [4]. In the present study, bilateral nodal disease was not a significant predictor of gastrostomy tube dependence and was not included in the multivariate regression. The lack of patientreported quality of life outcomes is another limitation.

\section{Conclusions}

This hypothesis-generating study shows that cSMG sparing radiotherapy may reduce post-treatment PEG dependence and dysphagia. We are currently conducting a prospective quality of life study to learn whether cSMG sparing is associated with improvement in patient-reported outcomes.

\section{Abbreviations}

CSMG: contralateral submandibular gland; CT: Computed tomography; CTCAE: Common Terminology Criteria for Adverse Events; IMRT: Intensitymodulated radiation therapy; PEG: Percutaneous endoscopic gastrostomy; PTV: Planning target volume; SMG: Submandibular gland

\section{Acknowledgements}

Not applicable.

Funding

Not applicable.

Availability of data and materials

The data from this study are not publicly available.

\section{Authors' contributions}

All authors contributed to study design. MG and UP wrote the manuscript. All authors revised the manuscript and then approved the final manuscript.

\section{Competing interests}

The authors declare that they have no competing interests.

Consent for publication

Not applicable.

Ethics approval and consent to participate

This retrospective study was approved by our Institutional Review Board, protocol \#7677.

\section{Author details}

'Department of Radiation Oncology, Stanford University, Stanford, CA, USA.

${ }^{2}$ Department of Radiation Oncology, University of Washington Medical Center, 1959 NE Pacific St Box 356043, Seattle, WA 98195, USA. 
Received: 18 August 2016 Accepted: 4 November 2016

Published online: 15 November 2016

\section{References}

1. Batth SS, Caudell JJ, Chen AM. Practical considerations in reducing swallowing dysfunction following concurrent chemoradiotherapy with intensity-modulated radiotherapy for head and neck cancer. Head Neck. 2014;36:291-8

2. $\quad$ Feng FY, Kim HM, Lyden TH, Haxer MJ, Worden FP, et al. Intensity-modulated chemoradiotherapy aiming to reduce dysphagia in patients with oropharyngeal cancer: clinical and functional results. J Clin Oncol. 2010;28:2732-8.

3. Eisbruch A, Kim HM, Feng FY, Lyden TH, Haxer MJ, et al. Chemo-IMRT of oropharyngeal cancer aiming to reduce dysphagia: swallowing organs late complication probabilities and dosimetric correlates. Int J Radiat Oncol Biol Phys. 2011;81(3):e93-9.

4. Setton J, Lee NY, Riaz N, Huang SH, Waldron J, et al. A multi-institution pooled analysis of gastrostomy tube dependence in patients with oropharyngeal cancer treated with definitive intensity-modulated radiotherapy. Cancer. 2015;121:294-301.

5. Christianen ME, Schilstra C, Beetz I, Muijs CT, Chouvalova O, et al. Predictive modelling for swallowing dysfunction after primary (chemo)radiation: results of a prospective observational study. Radiother Oncol. 2012;105:107-14.

6. Dirix P, Abbeel S, Vanstraelen B, Hermans R, Nuyts S. Dysphagia afte chemoradiotherapy for head-and-neck squamous cell carcinoma: doseeffect relationships for the swallowing structures. Int J Radiat Oncol Biol Phys. 2009;75:385-92.

7. Bhide SA, Gulliford S, Kazi R, El-Hariry I, Newbold K, et al. Correlation between dose to the pharyngeal constrictors and patient quality of life and late dysphagia following chemo-IMRT for head and neck cancer. Radiother Oncol. 2009:93:539-44.

8. Hamlet S, Faull J, Klein B, Aref A, Fontanesi J, et al. Mastication and swallowing in patients with postirradiation xerostomia. Int J Radiat Oncol Biol Phys. 1997:37:789-96.

9. Vainshtein JM, Samuels S, Tao Y, Lyden T, Haxer M, et al. Impact of xerostomia on dysphagia after chemotherapy-intensity-modulated radiotherapy for oropharyngeal cancer: prospective longitudinal study. Head Neck. 2016;38:E1605-12.

10. Mortensen HR, Jensen K, Aksglæde K, Behrens M, Grau C. Late dysphagia after IMRT for head and neck cancer and correlation with dose-volume parameters. Radiother Oncol. 2013;107:288-94.

11. Teguh DN, Levendag PC, Noever I, van Rooij P, Voet $P$, et al. Treatment techniques and site considerations regarding dysphagia-related quality of life in cancer of the oropharynx and nasopharynx. Int J Radiat Oncol Biol Phys. 2008;72:1119-27.

12. Nutting CM, Morden JP, Harrington KJ, Urbano TG, Bhide SA, et al. PARSPORT trial management group. Parotid-sparing intensity modulated versus conventional radiotherapy in head and neck cancer (PARSPORT): a phase 3 multicentre randomised controlled trial. Lancet Oncol. 2011;12:127-36.

13. Gensheimer MF, Liao JJ, Garden AS, Laramore GE, Parvathaneni U. Submandibular gland-sparing radiation therapy for locally advanced oropharyngeal squamous cell carcinoma: patterns of failure and xerostomia outcomes. Radiat Oncol. 2014:9:255

14. Tam M, Riaz N, Kannarunimit D, Pena AP, Schupak KD, Gelblum DY, et al. Sparing bilateral level IB in oropharyngeal ca and xerostomia outcomes. Am J Clin Oncol. 2015;38:343-7.

15. Tabak LA. In defense of the oral cavity: structure, biosynthesis, and function of salivary mucins. Annu Rev Physiol. 1995;57:547-64

16. Dantas RO, Kern MK, Massey BT, Dodds WJ, Kahrilas PJ, et al. Effect of swallowed bolus variables on oral and pharyngeal phases of swallowing. Am J Physiol Gastrointest Liver Physiol. 1990;258:G675-81.

17. Prinz JF, Lucas PW. An optimization model for mastication and swallowing in mammals. Proc R Soc Lond B. 1997;264:1715-21.

18. Roger-Leroi V, Mishellany-Dutour A, Woda A, Marchand M, Peyron MA Substantiation of an artificial saliva formulated for use in a masticatory apparatus. Odontostomatol Trop. 2012;35:5-14.

19. National Cancer Institute. Common terminology criteria for adverse events version 4.0. [https://evs.nci.nih.gov/ftp1/CTCAE/CTCAE_4.03_2010-06-14_ QuickReference_8.5x11.pdf]. Accessed 16 Mar 2016.
20. Christianen ME, Langendijk JA, Westerlaan HE, van de Water TA, Bijl HP. Delineation of organs at risk involved in swallowing for radiotherapy treatment planning. Radiother Oncol. 2011;101:394-402.

21. Little M, Schipper M, Feng FY, Vineberg K, Cornwall C, et al. Reducing xerostomia after chemo-IMRT for head-and-neck cancer: beyond sparing the parotid glands. Int J Radiat Oncol Biol Phys. 2012;83:1007-14.

22. Wopken K, Bijl HP, van der Schaaf A, van der Laan HP, Chouvalova O, et al. Development of a multivariable normal tissue complication probability (NTCP) model for tube feeding dependence after curative radiotherapy/ chemo-radiotherapy in head and neck cancer. Radiother Oncol. 2014;113:95-101.

23. Ronis DL, Duffy SA, Fowler KE, Khan MJ, Terrell JE. Changes in quality of life over 1 year in patients with head and neck cancer. Arch Otolaryngol Head Neck Surg. 2008;134:241-8.

24. Jensen K, Bonde Jensen A, Grau C. The relationship between observerbased toxicity scoring and patient assessed symptom severity after treatment for head and neck cancer. A correlative cross sectional study of the DAHANCA toxicity scoring system and the EORTC quality of life questionnaires. Radiother Oncol. 2006;78:298-305.

\section{Submit your next manuscript to BioMed Central and we will help you at every step:}

- We accept pre-submission inquiries

- Our selector tool helps you to find the most relevant journal

- We provide round the clock customer support

- Convenient online submission

- Thorough peer review

- Inclusion in PubMed and all major indexing services

- Maximum visibility for your research

Submit your manuscript at www.biomedcentral.com/submit
) Biomed Central 